\title{
DAWL: A Delayed-ACK Scheme for MAC-Level Performance Enhancement of Wireless LANs
}

\author{
DZMITRY KLIAZOVICH and FABRIZIO GRANELLI \\ DIT-University of Trento, Via Sommarive 14, I-38050 Trento, Italy
}

\begin{abstract}
The IEEE 802.11 MAC protocol provides a reliable link layer using Stop \& Wait ARQ. The cost for high reliability is the overhead due to acknowledgement packets in the direction opposite to the actual data flow. In this paper, the design of a new protocol as an enhancement of IEEE 802.11 is proposed, with the aim of reducing supplementary traffic overhead and increasing the bandwidth available for actual data transmission. The performance of the proposed protocol is evaluated through comparison with IEEE 802.11 as well as with a SSCOP-based protocol. Results underline significant advantages of the proposed protocol against existing ones, thus confirming the value and potentiality of the approach.
\end{abstract}

Keywords: wireless LAN, IEEE 802.11, delayed ACK, MAC-level optimization

\section{Introduction}

In the last few years, wireless communication has gained worldwide importance. In such an environment, where packets can be lost due to errors, collisions and hidden nodes, IEEE 802.11 standard [8] represents the leading MAC (Medium Access Control) solution for wireless local area networks (WLAN). 802.11 provides a reliable link layer by handling the packet delivery problems using a Stop\&Wait ARQ (Automatic Repeat Request) scheme. This means that each transmitted packet must be acknowledged before the next packet can be sent. In IEEE 802.11 the receiver of the packet must reply with a positive acknowledgement (PACK frame) to sender. The reception of this acknowledgement indicates successful frame transmission. If either the packet or its acknowledgement is lost, the sender of the packet will not receive any acknowledgement, and it will retransmit the packet after a certain timeout period (ACK_Timeout).

The advantages of this scheme are the high reliability of data delivery and the ease of implementation. However, such an ARQ scheme is as inefficient as any other Stop $\&$ Wait scheme, due to the idle time spent in waiting for the receiver acknowledgement after each transmission [2]. An experimental study of the IEEE 802.11 ARQ scheme is described in [4], where its weak points are described when the reliability of the link layer is not acceptable for higher layer protocols such as TCP.

Several studies were performed for improvement of the performance of the 802.11 logical link control via modification of its ARQ scheme. The alternative local area network protocol proposed in [3] and the Enhanced Retransmission Scheme [6] were designed to reduce the number of control frames used for single-packet delivery. A new SSCOP-based protocol was proposed in [7] for the improvement of the acknowledgement scheme, aimed at the reduction of overhead due to acknowledgements. This becomes possible by collecting the acknowledgement information on the receiver side and then sending it, using one control frame, after being polled by transmitter. This technique, known as Service Specific Connection Oriented Protocol (SSCOP), has already been implemented in ATM networks.

This paper presents a new approach to the reduction of the acknowledgement overhead for improvement of the throughput in wireless channels with different error rates. The proposed protocol, that can be considered as a modification of the original 802.11 standard, exploits the main concepts of the TCP Delayed-ACK scheme as well as a negative acknowledgement technique.

The rest of the paper is organized as follows. In Section 2 the proposed scheme is introduced in details, first by introducing the main concepts and features of the protocol and then by describing the modifications required to 802.11 in Section 3. Performance evaluation through simulation is presented in Section 4. Finally conclusions and outlines of future work on the topic are proposed in Sections 5 and 6.

\section{Description of the proposed method}

\subsection{General description}

The proposed protocol-Delayed-ACK for Wireless LANs (DAWL) - is a combination of TCP Delayed-ACK scheme and SSCOP-based protocols. The main concept behind the proposed method is that the receiver does not acknowledge the packets' delivery immediately, but it delays their acknowledgement. Assuming to have data going in the direction opposite to the actual flow of data, the acknowledgement can be sent together with data packet, therefore decreasing the overall packet delivery time. To this aim, Positive ACKnowledgements (PACK) are used to acknowledge the data packet delivery and Negative ACKnowledgements (NACK) to request retransmission of missing packets. 
In the following, in order to simplify the presentation of the proposed approach, we assume that there is only a single link between the transmitter and the receiver, because there is only one station allowed to transmit at certain moment of time. In case more than one transmitter is working at the same moment there will be a collision and transmission will incur in packet drops. This means that all transmitted packets are going continuously one-by-one. It is possible to detect packet losses by analyzing the order of sequence numbers of the received packets. When a missing packet is detected, the receiver sends a NACK message, mentioning the sequence number and the amount of missed packets to let the sender retransmit the missing packets. The packets for positive and negative acknowledgement are control packets of the MAC layer and they are to be transmitted after the Short Inter Frame Space (SIFS) time interval.

Figure 1 shows an example of the basic operations of DAWL protocol. The data packets Data (1.1) and Data (1.2) from Node 1 are received by Node 2 without acknowledgement, until there is a data packet Data (2.1). Then, PACK (1.2) is transmitted within such data packet in order to acknowledge the previously received frames. Since Node 2 has no additional data to send, acknowledgements to be sent to Node 1 are collected and when the PACKDelay timeout expires, Node 2 reports to Node 1 about successful reception of last frames (1.3), (1.4) and (1.5) by the transmission of PACK(1.5) control packet.

The main difference from IEEE 802.11 MAC protocol, like in SSCOP-based approach, is the elimination of ACK timeout and the reduction of the medium busy time, which is required for every data packet acknowledgement (except broadcast).

The difference with the SSCOP-based protocol presented in [7] is the elimination of control packets transmission such as STAT. In [7], in fact, after sending a specified amount of

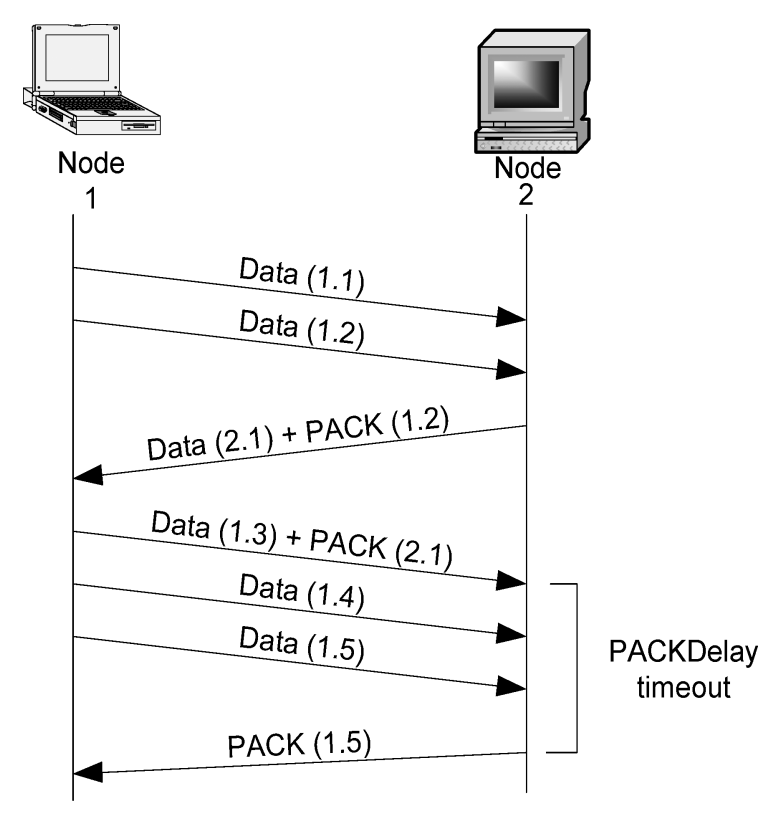

Figure 1. An example of the basic mode of operation in DAWL. data frames, the transmitter will wait for the STAT frame from the receiver. The STAT frame is a control frame of variable length which is to be sent after the SIFS interval, without medium reservation by Network Allocation Vector (NAV). Its elimination will decrease the probability of collision in the medium. In DAWL, on the contrary, acknowledgements go along with data packets since in most of the cases data packets are present in both directions.

\subsection{Error recovery using the DAWL protocol}

In case of operation in channels with errors (like wireless LANs), the DAWL protocol provides a fast error recovery mechanism to maintain the average throughput at a high level. Figure 2 displays an example of operation in different error scenarios. When the receiver detects the loss of data packets Data (1.3) and Data (1.4), it informs the sender by sending a retransmission request for the missed packets. It is possible to request more then one packet because NACK contains a sequence number and the amount of packets to be retransmitted. After the reception of NACK, the sender must retransmit the requested packets. In case there is no retransmission caused by NACK when it was lost, but there is a continuation of data flow, detected upon the arrival of the next data frame, the NACK request must be repeated (figure 2). After successful retransmission of the lost frames, the transmitter's data flow continues. If the sender does not receive a positive acknowledgement for the transmitted data within POLL timeout time, it will poll the receiver through transmission of POLL frame. Upon reception of the POLL frame, the receiver must immediately answer with PACK. The main differences from SSCOP-based protocol are:

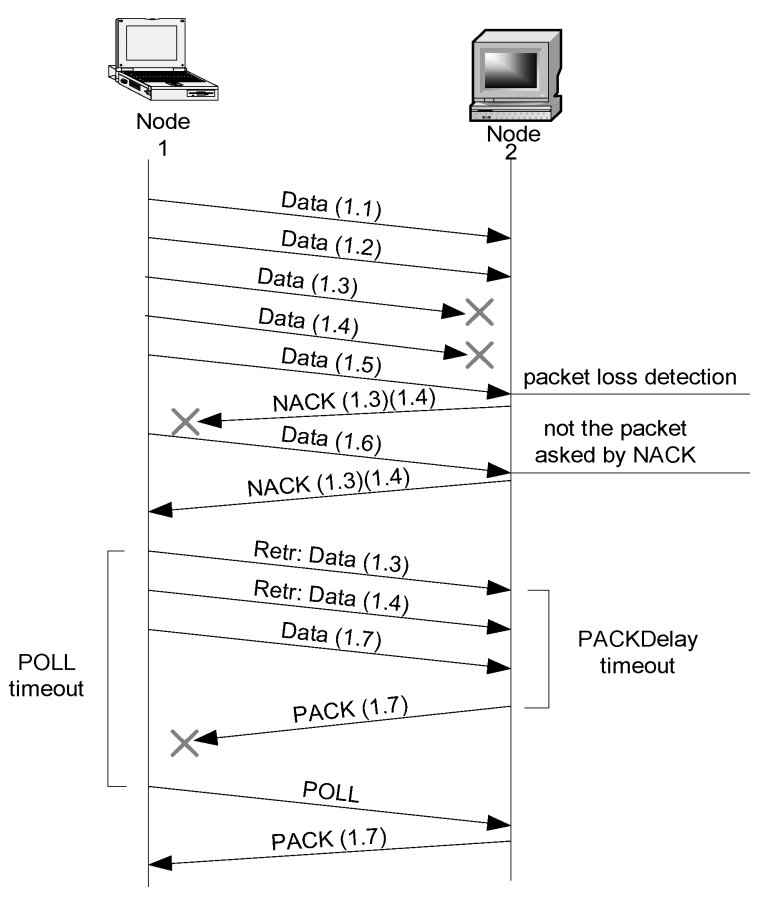

Figure 2. Examples of error events and recovery in DAWL. 


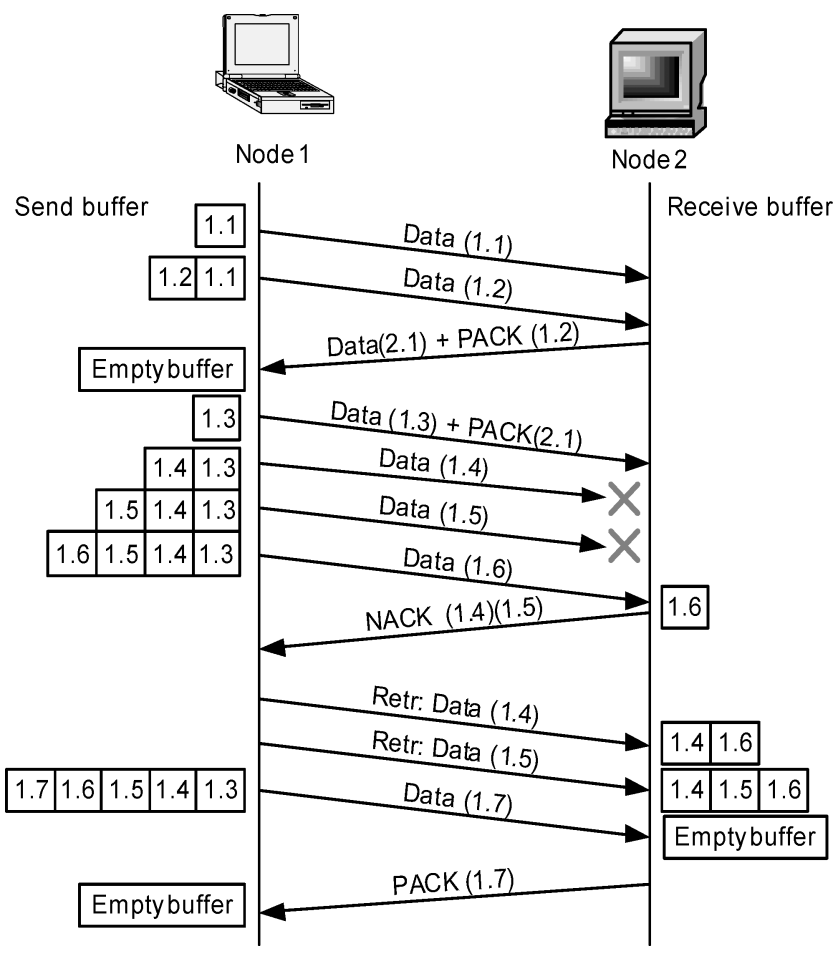

Figure 3. Buffer management in DAWL.

- the possibility of requesting retransmission of more than one frame at a time;

- the sender is not freezing the data flow while waiting for the acknowledgement for the sent packets;

- the retransmission of POLL using the SSCOP-based approach takes much more channel resources then retransmission of a POLL frame in the proposed protocol, because in the latter case it is just a control packet.
- The reader should note that the POLL frame in DAWL protocol will not appear in usual data exchange even with reasonable percentage of errors. It is mainly added to handle non-standard situations when there are no acknowledgements from the receiver for a long time.

\subsection{Timeouts}

The DAWL protocol provides a reliable link layer. However, some data packets and acknowledgements can be lost. POLL timeout is used to handle the situation of missing acknowledgements and its expiration causes generation of POLL frame by sender to get the status information from the receiver. This is the only situation in which a POLL frame can appear during packet exchange.

An additional timer, PACKDelay timeout, must be defined, that is similar to the case of TCP Delayed ACK. During this time a node deliberately delays sending PACK assuming it can send it along with the data. In case there is no data to send, it will send a standalone PACK.

Such timers should be carefully configured in order to trigger retransmission before higher layer timeouts occur. Furthermore, the POLL timeout value must be larger than PACKDelay timeout in order to eliminate the transmission of unnecessary POLL frames. The optimal value of the PACKDelay timeout depends on the current channel error rate. As a guideline, it is recommended to choose a timeout value that is enough for transmission of 3-4 transport layer frames each consisting of 1500 bytes.

\subsection{Buffer management}

All data packets to be sent must be placed in a buffer before transmission. They can be deleted from the buffer after the

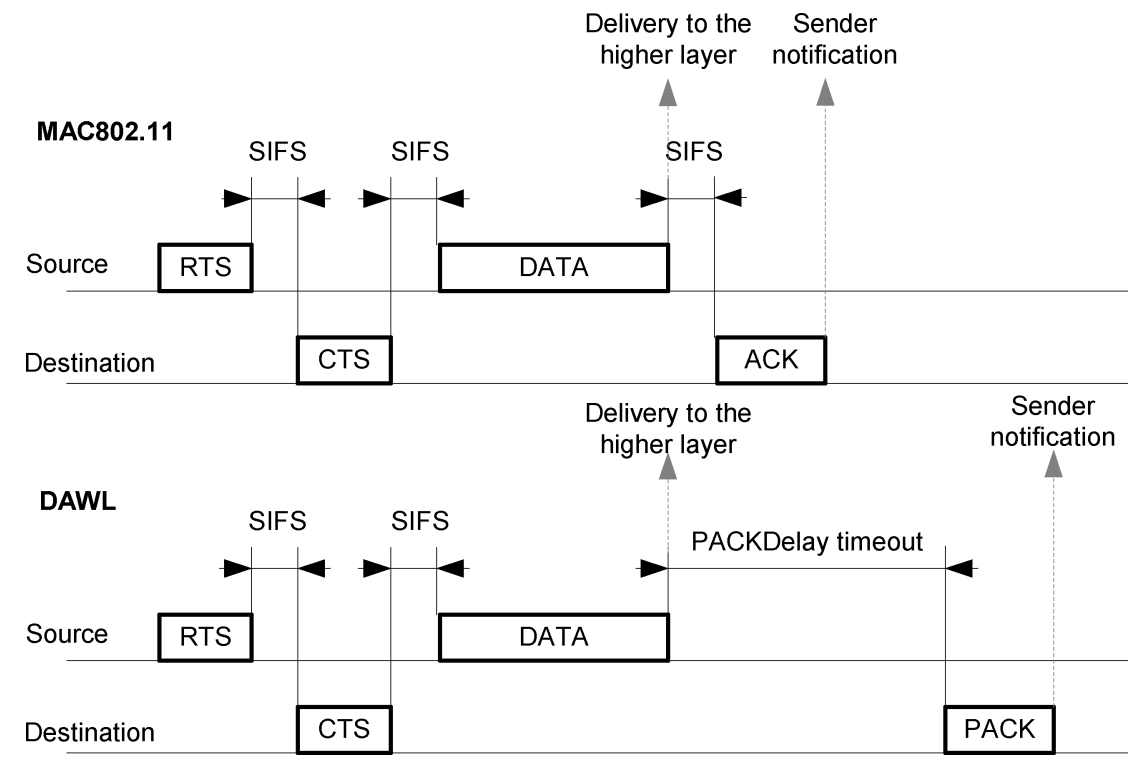

Figure 4. Packet delivery and sender notification events. 


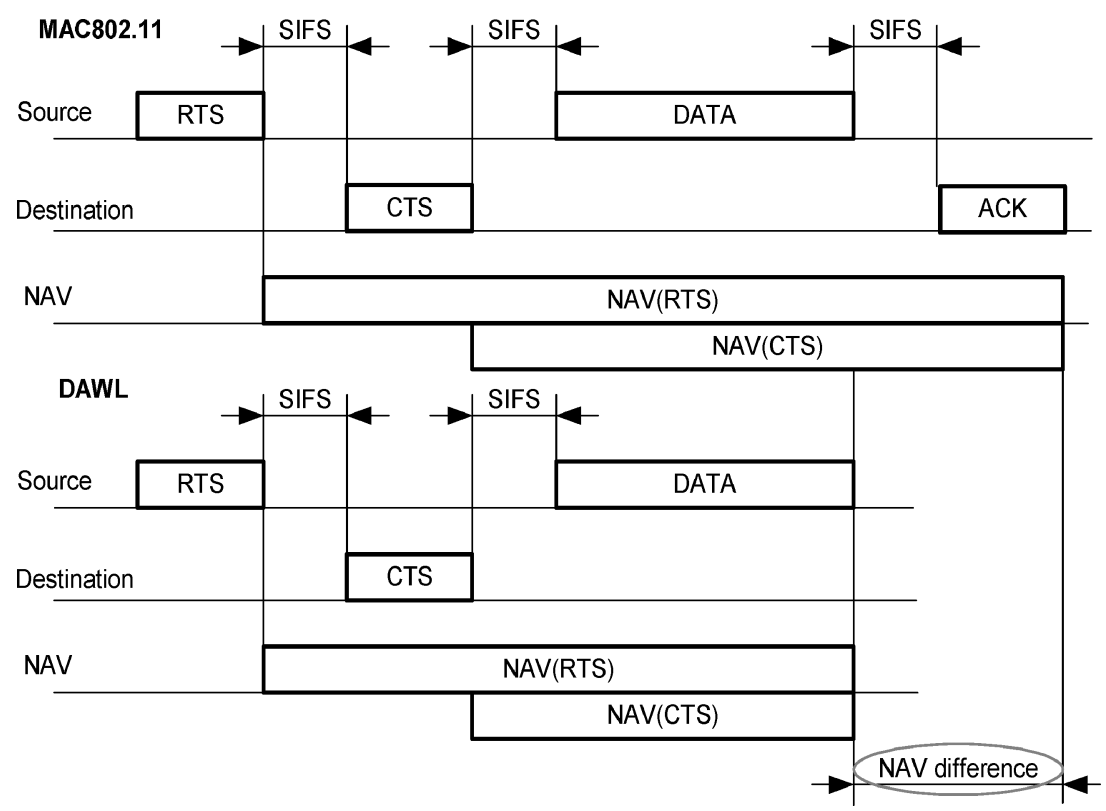

Figure 5. The difference between NAV calculation in the original 802.11 and DAWL protocols.

corresponding positive acknowledgements are received. On the receiver side, when a packet loss is detected, all frames from this time, until missing frames are retransmitted and successfully received, are to be put to the buffer. As it is shown in figure 3, frames 1.1 and 1.2 are buffered at the transmitter until a PACK is received within data packet 2.1. The receiver detects a frame loss when frame 1.6 is received, consequently it puts the frame into the buffer as well as retransmitted packets 1.4 and 1.5. Then, when frame 1.7 is received, the receiver releases the buffer. Finally, upon reception of the PACK standalone frame, the sender deletes successfully transmitted frames from the buffer.

\subsection{Packet delivery time and sender notification}

One of the most important parameters of a protocol functionality is the packet delivery time, especially in the case where control packets accompany data packet delivery.

In figure 4, a standalone higher layer packet transmission is considered in order to show the packet delivery time of DAWL protocol in comparison with MAC 802.11. Upon the DATA packet reception and its checksum verification for error detection, the receiver is able to decide about the successful reception of data and can pass the received data to the upper layer of the protocol stack. Since the timing of the RTS-CTS-DATA exchange is exactly the same in both cases, we can conclude that packet delivery time of DAWL and MAC 802.11 is exactly the same. As a result, we can infer that the modifications proposed in the DAWL protocol are completely transparent for the higher layer protocols operation such as TCP with its Round Trip Time (RTT) calculation algorithms.

The final stage of the packet delivery is the sender notification of the delivery status. The PACKDelay timeout in DAWL is bigger than SIFS in MAC. This generates a longer delay in the reply to a standalone DATA packet transmission (as in

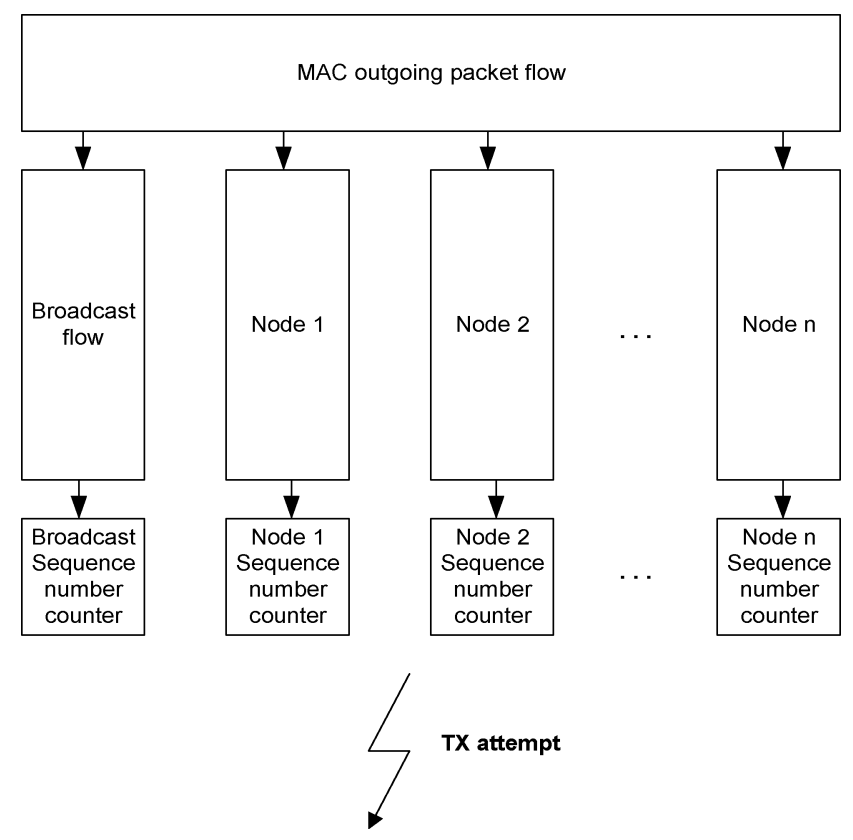

Figure 6. Sequence number management in DAWL.

figure 4), but it does not imply a insignificant impact on the data traffic since notification events are related to the release of the resources allocated for the transmission and for the data flow control mechanism.

\section{Proposed modifications of IEEE 802.11 MAC protocol}

\subsection{NAV modification}

The elimination of ACK frames in the data frame transmission scheme of 802.11 requires a modification in the NAV value 
Data + PACK packet:

\begin{tabular}{|c|c|c|c|c|c|c|c|c|c|} 
Octets: 2 & \multicolumn{1}{c}{6} & \multicolumn{2}{c}{6} & 2 & 6 & 2 & 2312 & 4 \\
\hline $\begin{array}{c}\text { Frame } \\
\text { Control }\end{array}$ & $\begin{array}{c}\text { Duration/l } \\
\mathrm{D}\end{array}$ & Address 1 & Address 2 & Address 3 & $\begin{array}{c}\text { Sequence } \\
\text { Control }\end{array}$ & $\begin{array}{c}\text { PACK } \\
\text { Sequence }\end{array}$ & Address 4 & $\begin{array}{c}\text { Frame } \\
\text { Body }\end{array}$ & FCS \\
\hline
\end{tabular}

Positive acknowledgement frame (PACK):

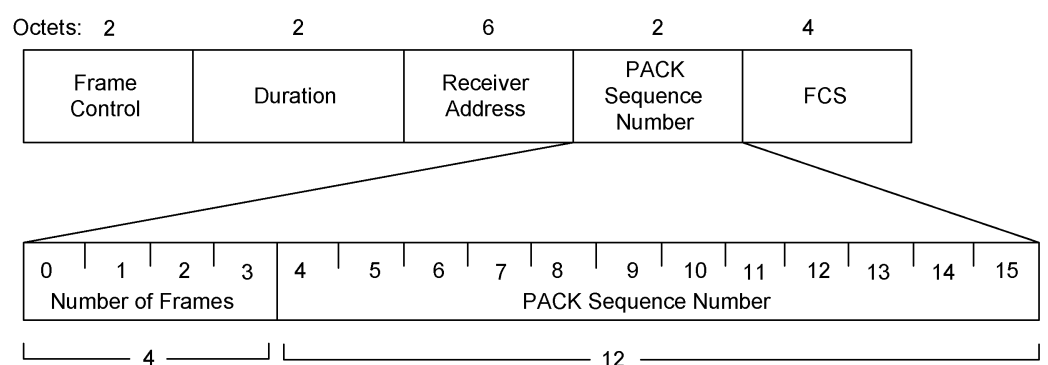

Negative acknowledgement frame (NACK):

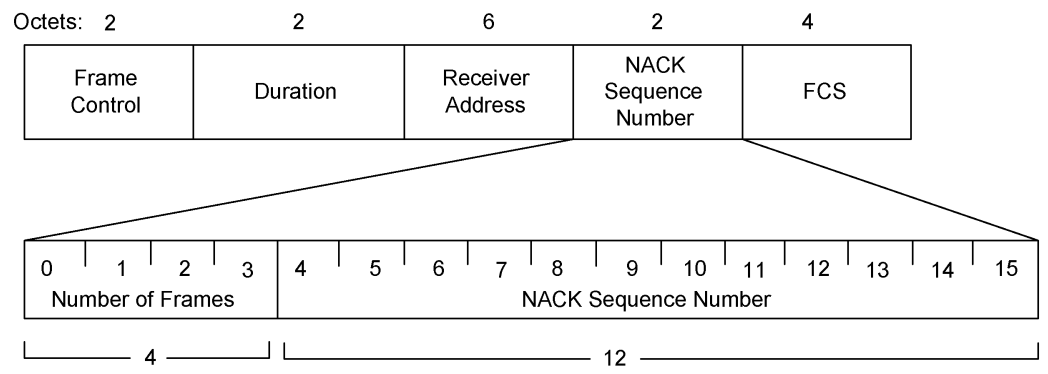

Negative and Positive acknowledgements frame (NACK + PACK):

\begin{tabular}{|c|c|c|c|c|c|} 
Octets: 2 & \multicolumn{2}{c}{2} & 2 & 2 & 4 \\
\hline \begin{tabular}{|l} 
Frame \\
Control
\end{tabular} & Duration & $\begin{array}{c}\text { Receiver } \\
\text { Address }\end{array}$ & $\begin{array}{c}\text { NACK } \\
\text { Sequence } \\
\text { Number }\end{array}$ & $\begin{array}{c}\text { PACK } \\
\text { Sequence } \\
\text { Number }\end{array}$ & FCS \\
\hline
\end{tabular}

POLL frame:

\begin{tabular}{|l|l|l|l|} 
Octets: 2 & \multicolumn{2}{c|}{6} & 4 \\
\hline $\begin{array}{l}\text { Frame } \\
\text { Control }\end{array}$ & Duration & $\begin{array}{l}\text { Receiver } \\
\text { Address }\end{array}$ & FCS \\
\hline
\end{tabular}

Figure 7. Modified frame formats in DAWL.

calculations (see figure 5). The modified NAV will not include the SIFS interval and ACK frame durations as shown in the figure 5, where the difference between 802.11 standard and DAWL in the calculation of NAV is underlined. POLL frame includes NAV for SIFS and PACK answer durations. The contention phase is still exactly the same as in MAC802.11.

\subsection{Sequence number management}

The IEEE 802.11 standard assigns a sequence number to every data packet: after transmission of the current packet, a sequence number counter (SNC) is increased modulo 4096.

Packet loss detection and the consequent retransmission of the lost packets in the DAWL protocol are based on the analysis of the order of the packet sequence numbers. In or- der to have an uninterrupted increase of sequence numbers in data exchange between two nodes, DAWL protocol needs to modify the original 802.11 scheme. Each node of the wireless network should have one SNC for broadcast packets and one SNC for each node to which the current node is making

Table 1

Frame type identifiers defined in the dawl protocol.

\begin{tabular}{lll}
\hline Frame type & Type & Subtype \\
\hline Data + PACK & 10 & 1000 \\
PACK & 01 & 1001 \\
NACK & 01 & 0110 \\
NACK + PACK & 01 & 1010 \\
POLL & 01 & 0010 \\
\hline
\end{tabular}




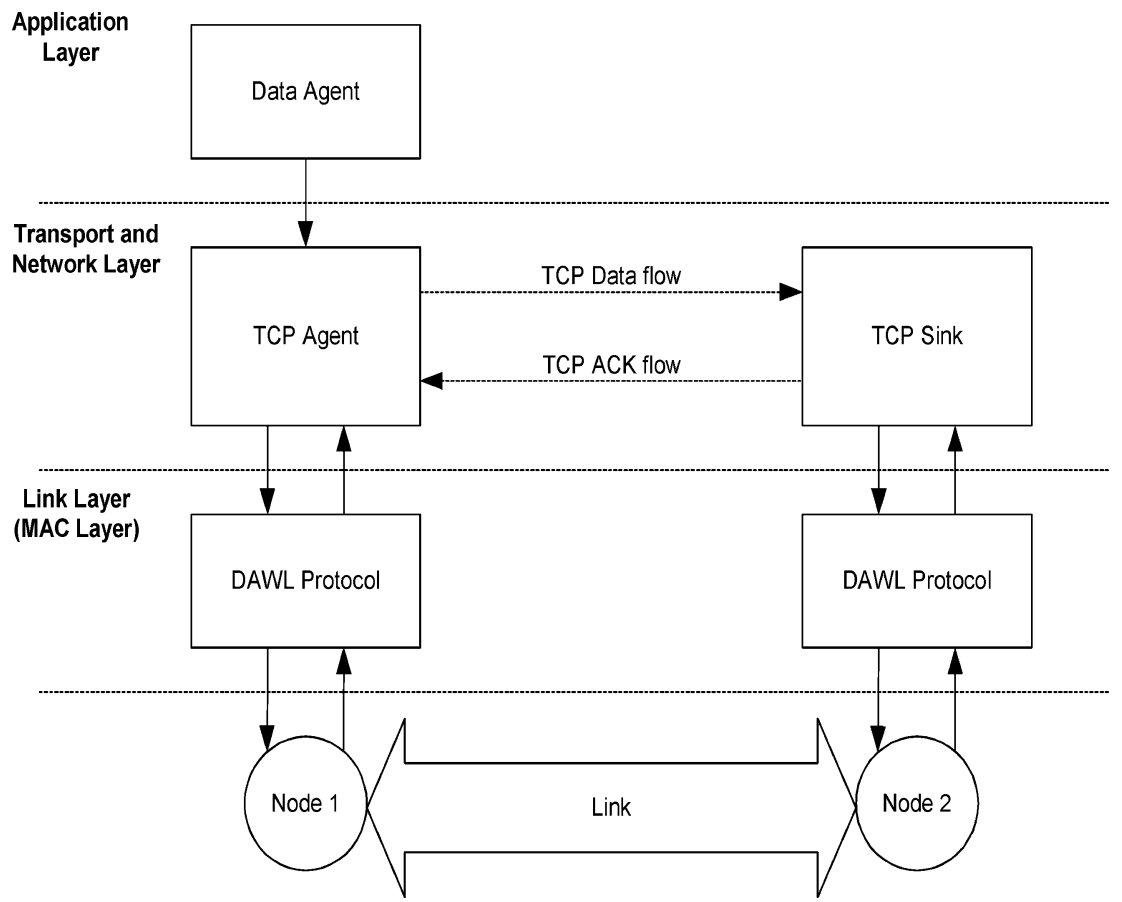

Figure 8. Protocol stack of two-node scenario model in ns-2 simulator.

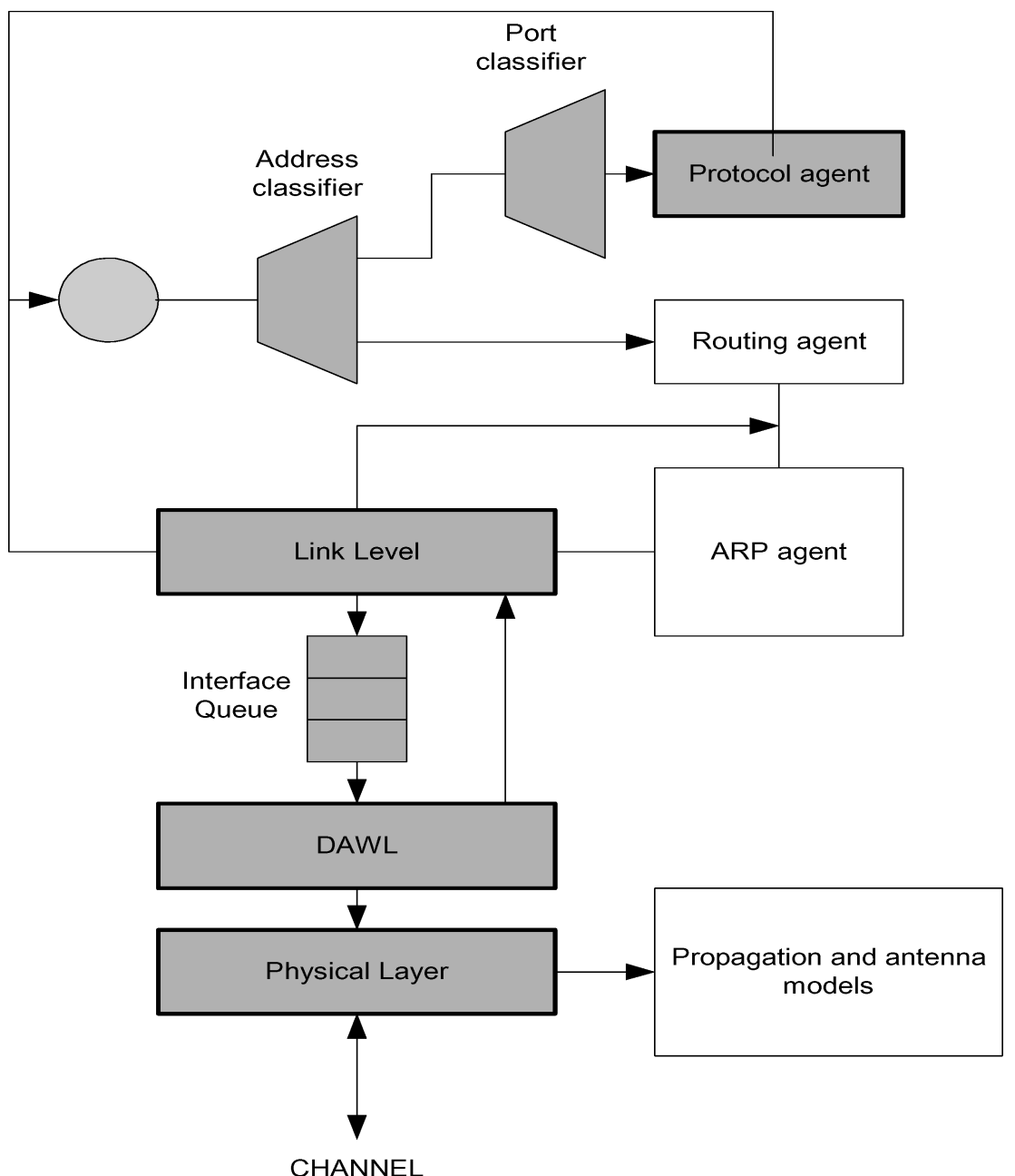

Figure 9. Ns Node structure in simulations. 
Table 2

Experimental results of tested protocols for TCP tahoe.

\begin{tabular}{llllllllll}
\hline & Error rate $(\%)$ & 0 & 3 & 5 & 7 & 9 & 11 & 13 \\
\hline Throughput & DAWL & 0.74 & 0.70 & 0.67 & 0.65 & 0.63 & 0.62 & 0.59 \\
(Mbits/s) & SSCOP & 0.73 & 0.69 & 0.66 & 0.61 & 0.58 & 0.56 & 0.53 & 0.46 \\
& MAC 802.11 & 0.70 & 0.64 & 0.58 & 0.52 & 0.48 & 0.44 & 0.37 & 0.32 \\
\hline
\end{tabular}

packet exchange at present moment. It means when a node has a necessity to send data to any other node it allocates a new SNC. When the data exchange with that node is finished it can release the allocated resources. A block scheme of such concept is shown in figure 6 .

\subsection{Frame formats}

In the DAWL protocol, five new packet types are introduced:

- Data + PACK frame, to carry acknowledgement information about the received frames;

- PACK frame, as a standalone packet;

- NACK frame, to inform the sender about a frame loss;

- NACK + PACK frame, as a combination of ACK and NACK frames, to decrease the number of small packets in the channel;

- POLL frame, to request receiver status information.

The framework of the new packets is presented in figure 7, while the newly defined frame types are in Table 1.

\section{Performance evaluation}

The performance of the proposed protocol is analyzed by simulating experiments using the ns-2 network simulator [5] Most of the results are achieved in grid topology where two static nodes are linked through a single TCP connection. One of them continuously sends data, while the other one only replies with TCP acknowledgements, which are considered as standard data frames for layer 2, where the proposed protocol operates. Figure 8 illustrates the protocol stack of the two nodes in the ns- 2 environment.

The DAWL protocol operation was also tested in a multiflow environment where there are N TCP flows produced by $2 * \mathrm{~N}$ nodes ( $\mathrm{N}$ ranging from 1 up to 10 ). This scenario showed conceptual similarity with the two-node scenario introduced above. Such experiments are not included due to space constraints.

For evaluating the actual performance bounds of the proposed strategy, it is important to have only the actual TCP data flow between the nodes. This is achieved by disabling routing, ARP protocol, connection establishment and slow start phases of TCP itself. The resulting ns-2 node structure is depicted in figure 9 , where only grey blocks are enabled.

The throughput of TCP connections is chosen as the main parameter for the performance analysis. Drawing on the con- siderations presented in the introduction of the paper, we decided to compare the proposed protocol with IEEE 802.11 standard [8] as well as with the SSCOP-based protocol presented in [7].

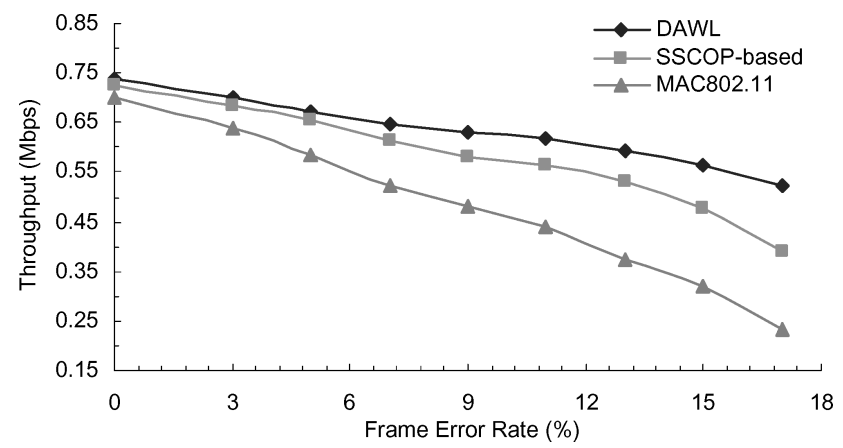

Figure 10. Performance comparison among 802.11, SSCOP-based and DAWL protocols in terms of throughput against channel error rate (TCP Tahoe).

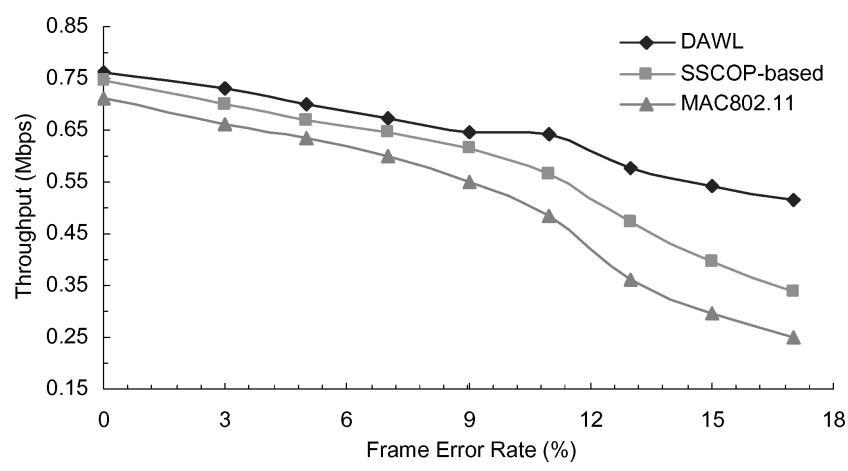

Figure 11. Performance comparison among 802.11, SSCOP-based and DAWL protocols in terms of throughput against channel error rate (TCP Reno with SACK and Delayed-ACK).

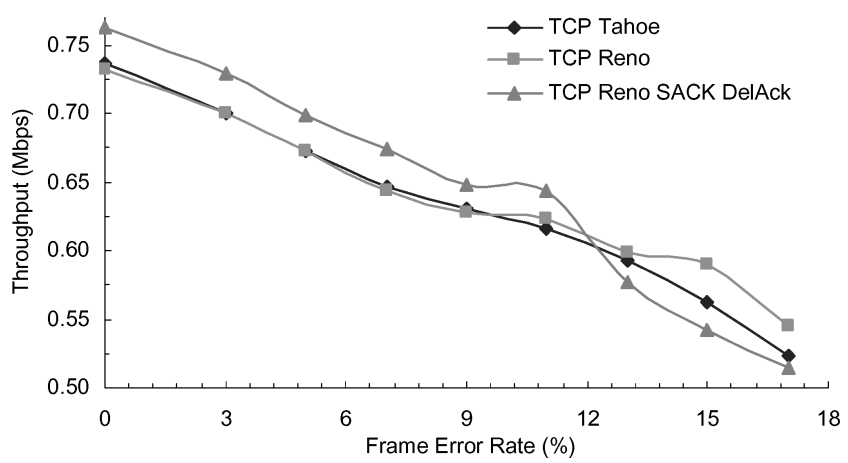

Figure 12. Comparison of the throughput of TCP Tahoe, Reno, SACK and Delayed-ACK schemes against the channel error rate. 

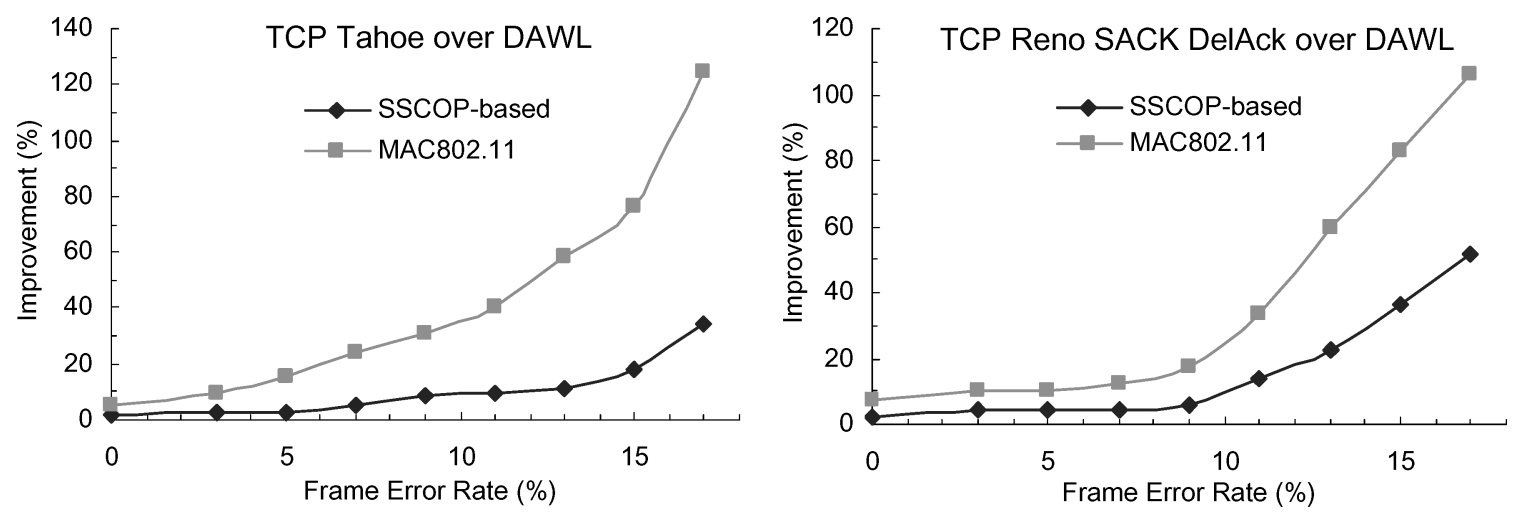

Figure 13. Improvement of DAWL versus 802.11 and SSCOP-based solutions for different TCP implementations.

TCP Tahoe and Reno are chosen for the evaluation, as the most common reference implementations for TCP [1]. The influence of Selective Acknowledgement (SACK) and Delayed Acknowledgement (Delayed-ACK) TCP schemes was investigated as well, in order to show the stability of DAWL protocol with reduced traffic in the direction opposite to actual data flow.

Figures 10 and 11 show simulation results. As the error rate increases, DAWL protocol has an increasing advantage compared with 802.11 MAC and SSCOP-based protocols. The main reason why the proposed protocol achieves higher throughput in comparison with SSCOP-based protocol is three-fold: (1) elimination of transmission of control frames, (2) improved retransmission algorithm and (3) absence of delay in data transmission while waiting for the acknowledgement of sent data frames. The difference with IEEE 802.11 is mainly due to the significant improvement in the acknowledgement scheme, which leads to faster data exchange between nodes. Some numerical values are presented in Table 2.

The comparison of the operation of DAWL protocol with different TCP implementations is shown in figure 12. Throughput values of TCP Tahoe and TCP Reno are quite close to each other, while SACK and Delayed-ACK schemes present improvement in low error rate operation $(<12 \%)$. In case of a higher error rate ( $>12 \%)$, the throughput becomes lower than the case of TCP Tahoe and Reno. Such throughput behavior is due to the impact of SACK and Delayed-ACK schemes of TCP. From these considerations, figure 12 underlines that DAWL has no influence on TCP operation, regardless of the specific implementation considered.

The percentage of the improvement of the DAWL protocol over the other approaches is shown in figure 13. The average throughput improvement of DAWL protocol in the interval from 0 to 10 percent of error rate of total channel bandwidth is around $4 \%$ as compared with the SSCOP-based protocol and $17 \%$ in the case of IEEE 802.11 MAC protocol. In case of higher channel error rate, the DAWL protocol provides a higher improvement. For the error interval from 10 to $17 \%$, in fact, the throughput improvements are in average $18.23 \%$ for the SSCOP-based protocol and $74.89 \%$ for IEEE 802.11 MAC.

Again, the advantage of the DAWL protocol is as bigger as channel error rate grows, due to the improvement in the management of packet losses.

\section{Conclusions}

This paper presented a new protocol, DAWL, as an enhancement of the IEEE 802.11 MAC, proposing the combination of the Delayed-ACK and negative acknowledgement techniques as a new alternative ARQ scheme for WLANs. The performance of the proposed protocol is evaluated by using different implementations of TCP and by comparing the results with standard IEEE 802.11 MAC as well as with a SSCOP-based protocol. Results underline significant advantages of the designed protocol in any channel error condition.

\section{Future work}

The DAWL protocol has obvious advantages in dual direction traffic exchange. The evaluation is carried out in scenario when there is a single TCP connection between two wireless nodes, thus creating a data flow in two directions (TCP data and ACK). Further work will deal with optimization of the parameters of the DAWL protocol in a multi-node errorprone wireless network and performance tests using different kinds of channel propagation models. It will also be useful to test the performance of the DAWL protocol in an environment characterized by a greater quantity of small packets with variable delays, like in a typical web session using the HTTP protocol.

At the present moment, we are also considering the possibility of producing an evaluation of the proposed protocol in real 802.11 wireless network environments by implementing DAWL protocol on a PC. 


\section{References}

[1] K. Fall and S. Floyd, Simulation-based comparisons of Tahoe, Reno, and SACK TCP, Computer Communication Review, 1996.

[2] H. Medeiros de Lima and O.C.M.B. Duarte, An Effective selective repeat ARQ strategy for high speed point-to-multipoint communications, Global Telecommunications Conference (GLOBECOM '96), 2 (1996) 1059-1063.

[3] B.E. Mullins, N.J. Davis and S.F. Midkiff, A wireless local area network protocol that improves throughput via adaptive control, IEEE International Conference on Communications (ICC'97), 3 (1997) 1427-1431.

[4] C. Schuler and M. Mateescu, "Performance Evaluation of ARQ Protocols for Realtime Services in IEEE 802.11 and Wireless ATM," 4th ACTS Mobile Communications Summit, Sorrento, Italy, 8-11 June 1999

[5] NS-2 simulator tool home page. http://www.isi.edu/nsnam/ns/, 2000.

[6] H.-L. Wang, J. Miao and J. Morris Chang, An enhanced IEEE 802.11 retransmission scheme, in: Proc. of IEEE Wireless Communication and Networking Conference (WCNC'03), (March 2003) vol. 1, pp. 66-71.

[7] Haoli Wang and A. Velayutham, An SSCOP-based link layer protocol for wireless LANs, IEEE Global Telecommunications Conference (GLOBECOM '03), 1 (2003) 453-457.

[8] Wireless LAN Medium Access Control (MAC) and Physical Layer (PHY) Specifications, IEEE 802.11 standard, 1997.

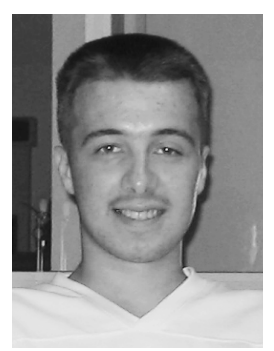

Dzmitry Kliazovich received his Masters degree in telecommunication science from Belarusian State University of Informatics and Radioelectronics in 2002. He is currently working towards the Ph.D. degree in University of Trento, Italy. His main research interest lies in wireless networking field with a focus on performance optimization and cross-layer design.

E-mail: klezovic@dit.unitn.it

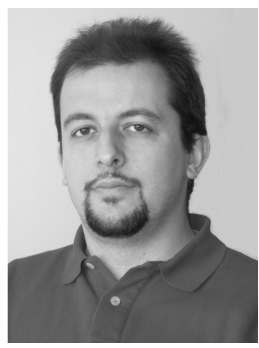

Fabrizio Granelli was born in Genoa in 1972 . He received the "Laurea" (M.Sc.) degree in Electronic Engineering from the University of Genoa, Italy, in 1997, with a thesis on video coding, awarded with the TELECOM Italy prize, and the Ph.D. degree in Electronic Engineering and Computer Science from the same university in 2001. Since 2000 he is carrying on his teaching activity as Assistant Professor at the Dept. of Information and Communication Technologies (DIT) of the University of Trento (Italy) within the B.Sc. and M.Sc. Degrees in Telecommunications Engineering.

The research interests of Dr. Granelli are mainly focused on networking, with particular attention to network modeling and performance evaluation, wireless networks, access control, and next-generation telecommunication networks.

He is author of more than 30 refereed papers, published in several international journals and conferences.

Dr. Granelli is member of the IEEE Committee on "Communication Systems Integration and Modeling" (CSIM) and of the Technical Programme Committee of the "QoS and Performance Evaluation Symposium" of the International Conference on Communications (ICC 2003 and ICC 2004).

E-mail: granelli@dit.unitn.it 